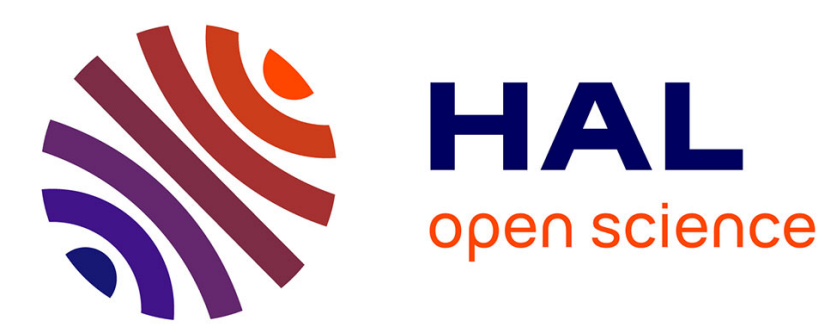

\title{
Exclusive production of pion and kaon meson pairs in two photon collisions at LEP
}

A. Heister, S. Schael, R. Barate, R. Bruneliere, I. de Bonis, D. Decamp, C. Goy, S. Jezequel, J.P. Lees, F. Martin, et al.

\section{- To cite this version:}

A. Heister, S. Schael, R. Barate, R. Bruneliere, I. de Bonis, et al.. Exclusive production of pion and kaon meson pairs in two photon collisions at LEP. Physics Letters B, 2003, 569, pp.140-150. in2p3-00014001

\author{
HAL Id: in2p3-00014001 \\ https://hal.in2p3.fr/in2p3-00014001
}

Submitted on 19 Sep 2003

HAL is a multi-disciplinary open access archive for the deposit and dissemination of scientific research documents, whether they are published or not. The documents may come from teaching and research institutions in France or abroad, or from public or private research centers.
L'archive ouverte pluridisciplinaire $\mathbf{H A L}$, est destinée au dépôt et à la diffusion de documents scientifiques de niveau recherche, publiés ou non, émanant des établissements d'enseignement et de recherche français ou étrangers, des laboratoires publics ou privés. 
EUROPEAN ORGANISATION FOR NUCLEAR RESEARCH

CERN-EP/2003-030

20 May 2003

\title{
Exclusive Production of Pion and Kaon Meson Pairs in Two Photon Collisions at LEP
}

The ALEPH Collaboration*

\begin{abstract}
Exclusive production of $\pi$ and $\mathrm{K}$ meson pairs in two photon collisions is measured with ALEPH data collected between 1992 and 2000. Cross sections are presented as a function of $\cos \theta^{*}$ and invariant mass, for $\left|\cos \theta^{*}\right|<0.6$ and invariant masses between 2.0 and $6.0 \mathrm{GeV} / c^{2}\left(2.25\right.$ and $\left.4.0 \mathrm{GeV} / c^{2}\right)$ for pions (kaons). The shape of the distributions are found to be well described by QCD predictions but the data have a significantly higher normalisation.
\end{abstract}

Submitted to Physics Letters B

${ }^{*}$ See next page for the list of authors 


\section{The ALEPH Collaboration}

A. Heister, S. Schael

Physikalisches Institut das RWTH-Aachen, D-52056 Aachen, Germany

R. Barate, R. Brunelière, I. De Bonis, D. Decamp, C. Goy, S. Jezequel, J.-P. Lees, F. Martin, E. Merle, M.-N. Minard, B. Pietrzyk, B. Trocmé

Laboratoire de Physique des Particules (LAPP), IN ${ }^{2} P^{3}$-CNRS, F-74019 Annecy-le-Vieux Cedex, France

S. Bravo, M.P. Casado, M. Chmeissani, J.M. Crespo, E. Fernandez, M. Fernandez-Bosman, Ll. Garrido, ${ }^{15}$ M. Martinez, A. Pacheco, H. Ruiz

Institut de Física d'Altes Energies, Universitat Autònoma de Barcelona, E-08193 Bellaterra (Barcelona), Spain ${ }^{7}$

A. Colaleo, D. Creanza, N. De Filippis, M. de Palma, G. Iaselli, G. Maggi, M. Maggi, S. Nuzzo, A. Ranieri, G. Raso, ${ }^{24}$ F. Ruggieri, G. Selvaggi, L. Silvestris, P. Tempesta, A. Tricomi,${ }^{3}$ G. Zito

Dipartimento di Fisica, INFN Sezione di Bari, I-70126 Bari, Italy

X. Huang, J. Lin, Q. Ouyang, T. Wang, Y. Xie, R. Xu, S. Xue, J. Zhang, L. Zhang, W. Zhao

Institute of High Energy Physics, Academia Sinica, Beijing, The People's Republic of China ${ }^{8}$

D. Abbaneo, T. Barklow, ${ }^{26}$ O. Buchmüller, ${ }^{26}$ M. Cattaneo, B. Clerbaux, ${ }^{23}$ H. Drevermann, R.W. Forty, M. Frank, F. Gianotti, J.B. Hansen, J. Harvey, D.E. Hutchcroft, ${ }^{30}$, P. Janot, B. Jost, M. Kado, ${ }^{2}$ P. Mato, A. Moutoussi, F. Ranjard, L. Rolandi, D. Schlatter, G. Sguazzoni, F. Teubert, A. Valassi, I. Videau

European Laboratory for Particle Physics (CERN), CH-1211 Geneva 23, Switzerland

F. Badaud, S. Dessagne, A. Falvard, ${ }^{20}$ D. Fayolle, P. Gay, J. Jousset, B. Michel, S. Monteil, D. Pallin, J.M. Pascolo, P. Perret

Laboratoire de Physique Corpusculaire, Université Blaise Pascal, $I N^{2} P^{3}$-CNRS, Clermont-Ferrand, F-63177 Aubière, France

J.D. Hansen, J.R. Hansen, P.H. Hansen, A.C. Kraan, B.S. Nilsson

Niels Bohr Institute, 2100 Copenhagen, DK-Denmark ${ }^{9}$

A. Kyriakis, C. Markou, E. Simopoulou, A. Vayaki, K. Zachariadou

Nuclear Research Center Demokritos (NRCD), GR-15310 Attiki, Greece

A. Blondel, ${ }^{12}$ J.-C. Brient, F. Machefert, A. Rougé, H. Videau 
Laoratoire Leprince-Ringuet, Ecole Polytechnique, $I N^{2} P^{3}$-CNRS, F-91128 Palaiseau Cedex, France

V. Ciulli, E. Focardi, G. Parrini

Dipartimento di Fisica, Università di Firenze, INFN Sezione di Firenze, I-50125 Firenze, Italy

A. Antonelli, M. Antonelli, G. Bencivenni, F. Bossi, G. Capon, F. Cerutti, V. Chiarella,

P. Laurelli, G. Mannocchi, ${ }^{5}$ G.P. Murtas, L. Passalacqua

Laboratori Nazionali dell'INFN (LNF-INFN), I-00044 Frascati, Italy

J. Kennedy, J.G. Lynch, P. Negus, V. O’Shea, A.S. Thompson

Department of Physics and Astronomy, University of Glasgow, Glasgow G12 8QQ,United Kingdom ${ }^{10}$

S. Wasserbaech

Utah Valley State College, Orem, UT 84058, U.S.A.

R. Cavanaugh, ${ }^{4}$ S. Dhamotharan, ${ }^{21}$ C. Geweniger, P. Hanke, V. Hepp, E.E. Kluge,

A. Putzer, H. Stenzel, K. Tittel, M. Wunsch ${ }^{19}$

Kirchhoff-Institut für Physik, Universität Heidelberg, D-69120 Heidelberg, Germany ${ }^{16}$

R. Beuselinck, W. Cameron, G. Davies, P.J. Dornan, M. Girone, ${ }^{1}$ R.D. Hill, N. Marinelli,

J. Nowell, S.A. Rutherford, J.K. Sedgbeer, J.C. Thompson, ${ }^{14}$ R. White

Department of Physics, Imperial College, London SW7 2BZ, United Kingdom ${ }^{10}$

V.M. Ghete, P. Girtler, E. Kneringer, D. Kuhn, G. Rudolph

Institut für Experimentalphysik, Universität Innsbruck, A-6020 Innsbruck, Austria ${ }^{18}$

E. Bouhova-Thacker, C.K. Bowdery, D.P. Clarke, G. Ellis, A.J. Finch, F. Foster,

G. Hughes, R.W.L. Jones, M.R. Pearson, N.A. Robertson, M. Smizanska

Department of Physics, University of Lancaster, Lancaster LA1 4YB, United Kingdom ${ }^{10}$

O. van der Aa, C. Delaere, ${ }^{28}$ G.Leibenguth, ${ }^{31}$ V. Lemaitre ${ }^{29}$

Institut de Physique Nucléaire, Département de Physique, Université Catholique de Louvain, 1348 Louvain-la-Neuve, Belgium

U. Blumenschein, F. Hölldorfer, K. Jakobs, F. Kayser, K. Kleinknecht, A.-S. Müller,

B. Renk, H.-G. Sander, S. Schmeling, H. Wachsmuth, C. Zeitnitz, T. Ziegler

Institut für Physik, Universität Mainz, D-55099 Mainz, Germany ${ }^{16}$

A. Bonissent, P. Coyle, C. Curtil, A. Ealet, D. Fouchez, P. Payre, A. Tilquin

Centre de Physique des Particules de Marseille, Univ Méditerranée, $I N^{2} P^{3}$-CNRS, F-13288 Marseille, France

F. Ragusa

Dipartimento di Fisica, Università di Milano e INFN Sezione di Milano, I-20133 Milano, Italy.

A. David, H. Dietl, G. Ganis, ${ }^{27}$ K. Hüttmann, G. Lütjens, W. Männer, H.-G. Moser, 
R. Settles, M. Villegas, G. Wolf

Max-Planck-Institut für Physik, Werner-Heisenberg-Institut, D-80805 München, Germany ${ }^{16}$

J. Boucrot, O. Callot, M. Davier, L. Duflot, J.-F. Grivaz, Ph. Heusse, A. Jacholkowska, ${ }^{6}$

L. Serin, J.-J. Veillet

Laboratoire de l'Accélérateur Linéaire, Université de Paris-Sud, $I N^{2} P^{3}$-CNRS, F-91898 Orsay Cedex, France

P. Azzurri, G. Bagliesi, T. Boccali, L. Foà, A. Giammanco, A. Giassi, F. Ligabue,

A. Messineo, F. Palla, G. Sanguinetti, A. Sciabà, P. Spagnolo R. Tenchini A. Venturi

P.G. Verdini

Dipartimento di Fisica dell'Università, INFN Sezione di Pisa, e Scuola Normale Superiore, I-56010 Pisa, Italy

O. Awunor, G.A. Blair, G. Cowan, A. Garcia-Bellido, M.G. Green, L.T. Jones, T. Medcalf,

A. Misiejuk, J.A. Strong, P. Teixeira-Dias

Department of Physics, Royal Holloway \& Bedford New College, University of London, Egham, Surrey TW20 OEX, United Kingdom ${ }^{10}$

R.W. Clifft, T.R. Edgecock, P.R. Norton, I.R. Tomalin, J.J. Ward

Particle Physics Dept., Rutherford Appleton Laboratory, Chilton, Didcot, Oxon OX11 OQX, United Kingdom ${ }^{10}$

B. Bloch-Devaux, D. Boumediene, P. Colas, B. Fabbro, E. Lançon, M.-C. Lemaire,

E. Locci, P. Perez, J. Rander, B. Tuchming, B. Vallage

CEA, DAPNIA/Service de Physique des Particules, CE-Saclay, F-91191 Gif-sur-Yvette Cedex, France $^{17}$

A.M. Litke, G. Taylor

Institute for Particle Physics, University of California at Santa Cruz, Santa Cruz, CA 95064, USA ${ }^{22}$

C.N. Booth, S. Cartwright, F. Combley, ${ }^{25}$ P.N. Hodgson, M. Lehto, L.F. Thompson

Department of Physics, University of Sheffield, Sheffield S3 7RH, United Kingdom ${ }^{10}$

A. Böhrer, S. Brandt, C. Grupen, J. Hess, A. Ngac, G. Prange

Fachbereich Physik, Universität Siegen, D-57068 Siegen, Germany ${ }^{16}$

C. Borean, G. Giannini

Dipartimento di Fisica, Università di Trieste e INFN Sezione di Trieste, I-34127 Trieste, Italy

H. He, J. Putz, J. Rothberg

Experimental Elementary Particle Physics, University of Washington, Seattle, WA 98195 U.S.A.

S.R. Armstrong, K. Berkelman, K. Cranmer, D.P.S. Ferguson, Y. Gao, ${ }^{13}$ S. González,

O.J. Hayes, H. Hu, S. Jin, J. Kile, P.A. McNamara III, J. Nielsen, Y.B. Pan,

J.H. von Wimmersperg-Toeller, W. Wiedenmann, J. Wu, Sau Lan Wu, X. Wu,

G. Zobernig

Department of Physics, University of Wisconsin, Madison, WI 53706, USA ${ }^{11}$ 
G. Dissertori

Institute for Particle Physics, ETH Hönggerberg, 8093 Zürich, Switzerland.

\footnotetext{
${ }^{1}$ Also at CERN, 1211 Geneva 23, Switzerland.

${ }^{2}$ Now at Fermilab, PO Box 500, MS 352, Batavia, IL 60510, USA

${ }^{3}$ Also at Dipartimento di Fisica di Catania and INFN Sezione di Catania, 95129 Catania, Italy.

${ }^{4}$ Now at University of Florida, Department of Physics, Gainesville, Florida 32611-8440, USA

${ }^{5}$ Also Istituto di Cosmo-Geofisica del C.N.R., Torino, Italy.

${ }^{6}$ Also at Groupe d'Astroparticules de Montpellier, Université de Montpellier II, 34095, Montpellier, France.

${ }^{7}$ Supported by CICYT, Spain.

${ }^{8}$ Supported by the National Science Foundation of China.

${ }^{9}$ Supported by the Danish Natural Science Research Council.

${ }^{10}$ Supported by the UK Particle Physics and Astronomy Research Council.

${ }^{11}$ Supported by the US Department of Energy, grant DE-FG0295-ER40896.

${ }^{12}$ Now at Departement de Physique Corpusculaire, Université de Genève, 1211 Genève 4, Switzerland.

${ }^{13}$ Also at Department of Physics, Tsinghua University, Beijing, The People's Republic of China.

${ }^{14}$ Supported by the Leverhulme Trust.

${ }^{15}$ Permanent address: Universitat de Barcelona, 08208 Barcelona, Spain.

${ }^{16}$ Supported by Bundesministerium für Bildung und Forschung, Germany.

${ }^{17}$ Supported by the Direction des Sciences de la Matière, C.E.A.

${ }^{18}$ Supported by the Austrian Ministry for Science and Transport.

${ }^{19}$ Now at SAP AG, 69185 Walldorf, Germany

${ }^{20}$ Now at Groupe d' Astroparticules de Montpellier, Université de Montpellier II, 34095 Montpellier, France.

${ }^{21}$ Now at BNP Paribas, 60325 Frankfurt am Mainz, Germany

${ }^{22}$ Supported by the US Department of Energy, grant DE-FG03-92ER40689.

${ }^{23}$ Now at Institut Inter-universitaire des hautes Energies (IIHE), CP 230, Université Libre de Bruxelles, 1050 Bruxelles, Belgique

${ }^{24}$ Also at Dipartimento di Fisica e Tecnologie Relative, Università di Palermo, Palermo, Italy.

${ }^{25}$ Deceased.

${ }^{26}$ Now at SLAC, Stanford, CA 94309, U.S.A

${ }^{27}$ Now at INFN Sezione di Roma II, Dipartimento di Fisica, Università di Roma Tor Vergata, 00133 Roma, Italy.

${ }^{28}$ Research Fellow of the Belgium FNRS

${ }^{29}$ Research Associate of the Belgium FNRS

${ }^{30}$ Now at Liverpool University, Liverpool L69 7ZE, United Kingdom

${ }^{31}$ Supported by the Federal Office for Scientific, Technical and Cultural Affairs through the Interuniversity Attraction Pole P $5 / 27$
} 


\section{Introduction}

The study of exclusive meson and baryon pair production has long been recognised as a potentially important testing ground of QCD [1, 2, 3, 4]. The calculation factorizes into a hard scattering amplitude which at sufficiently large momentum transfers is calculable in QCD, and a hadron distribution amplitude $\phi_{\mathrm{M}}(x, Q)$. As this distribution amplitude is expected to be process independent, measurements from many different reactions will be complementary. Measurements in photon photon collisions are important as they are the simplest calculable large-angle exclusive hadronic scattering reactions.

In [2] it is shown that the leading order QCD result can be expressed approximately in terms of the cross section for the two photon production of muon pairs:

$$
\frac{\mathrm{d} \sigma}{\mathrm{d} \cos \theta^{*}}\left(\gamma \gamma \rightarrow \mathrm{M}^{+} \mathrm{M}^{-}\right) \sim \frac{4\left|F_{\mathrm{M}}\left(W_{\gamma \gamma}^{2}\right)\right|^{2}}{1-\cos ^{4} \theta^{*}} \frac{\mathrm{d} \sigma}{\mathrm{d} \cos \theta^{*}}\left(\gamma \gamma \rightarrow \mu^{+} \mu^{-}\right)
$$

The angle $\cos \theta^{*}$ is the scattering angle of one of the two charged particles $\mathrm{M}$ with respect to the $\gamma \gamma$ direction, calculated in the $\gamma \gamma$ center of mass frame. The overall angular dependence of Eq. 1 is $\sin ^{-4} \theta^{*}$ when one folds in the muon pair cross section. The meson form factor $F_{\mathrm{M}}$ depends on the strong coupling constant $\alpha_{s}$, the $\gamma \gamma$ invariant mass $W_{\gamma \gamma}$, and the pseudoscalar decay constant $f_{\mathrm{M}}$.

This letter describes a measurement of the exclusive pion and kaon pair production in two photon collisions using the data collected by the ALEPH experiment at LEP. The absolute rate and the shape of the $\cos \theta^{*}$ distributions are compared to QCD calculations and previous experiments at lower energies.

\section{ALEPH Detector}

The ALEPH detector has been described in detail elsewhere [5]. Critical to this analysis is the ability to accurately measure and identify charged particles. These are detected in a large time projection chamber (TPC) supplemented by information from the inner tracking chamber (ITC) which is a cylindrical drift chamber sitting inside the TPC, and a two-layer silicon strip vertex detector (VDET) which surrounds the beam pipe close to the interaction point. These tracking detectors are placed inside a $1.5 \mathrm{~T}$ axial magnetic field provided by a superconducting solenoidal coil. Charged particle transverse momenta are measured with a resolution of $\delta p_{\mathrm{t}} / p_{\mathrm{t}}=$ 
$6 \times 10^{-4} p_{\mathrm{t}} \oplus 0.005\left(p_{\mathrm{t}}\right.$ in $\left.\mathrm{GeV} / c\right)$. In addition to measuring momenta, the TPC provides $\mathrm{d} E / \mathrm{d} x$ information which allows particle identification to be performed. Surrounding the solenoid and TPC is the electromagnetic calorimeter (ECAL) whose primary purpose is the identification and measurement of electromagnetic clusters produced by photons and electrons. It is a lead/proportional-tube sampling calorimeter segmented in $0.9^{\circ} \times 0.9^{\circ}$ projective towers read out in three sections in depth. It has a total thickness of 22 radiation lengths and a relative energy resolution of $0.18 / \sqrt{E}+0.009$, (E in $\mathrm{GeV}$ ). In this analysis it is mainly used as a veto against events containing electrons or photons which are readily identified by the longitudinal and transverse structure of their showers. Outside the ECAL lies the iron return yoke for the magnet which is instrumented with 23 layers of streamer tubes to form the hadron calorimeter (HCAL). It has a relative energy resolution for hadrons of $0.85 / \sqrt{E}(\mathrm{E}$ in $\mathrm{GeV})$. The outermost detectors of ALEPH are the muon chambers which consist of two double-layers of streamer tubes. The HCAL and muon chamber information is combined to identify muons which are a major potential background in this analysis. Close to the beam pipe at a position $3 \mathrm{~m}$ either side of the interaction point are two luminosity calorimeters, the LCAL and SiCAL, which are electromagnetic calorimeters specifically designed to measure the luminosity via Bhabha scattering. In this analysis they are used to veto events which contain significant energy deposits at small angles.

The information from the tracking detectors and the calorimeters are combined in an energy-flow algorithm [5]. For each event, the algorithm provides a set of charged and neutral reconstructed particles, called energyflow objects.

\section{Selection of Exclusively Produced Pairs of Charged Particles}

The data for this analysis are selected from $837.5 \mathrm{pb}^{-1}$ collected between 1992 and 2000. During this period the LEP centre of mass energy ranged from 88 to $209 \mathrm{GeV}$. The initial selection obtains events with two charged tracks exclusively produced by the $\gamma \gamma$ process. Events were required to have:

- two charged tracks, each of which has $|\cos \theta|<0.93, p_{\mathrm{t}}>1.0 \mathrm{GeV} / c$, at least 5 TPC hits and at least 6 ITC hits;

- a reconstructed vertex in a cylinder with its axis $8 \mathrm{~cm}$ either side of the interaction point along the beam direction and with a radius of $3 \mathrm{~cm}$; 
- no net charge;

- $\Sigma \mathbf{p}_{\mathrm{t}}<0.1 \mathrm{GeV} / c$

- no other energy flow objects apart from the two charged tracks;

- invariant mass of charged tracks (assuming pion mass) between 2 and $50 \mathrm{GeV} / c^{2}$;

- the back-to-back trigger set, as described in section 5.

\section{Selection of $\pi$ and K Pairs}

The purpose of this step is to select as pure a sample as possible of pion and kaon pairs. First, any event containing a track identified as an electron or as a muon was rejected. Electrons were identified using the standard ALEPH method [5]. For muons the standard identification was supplemented by the procedure described in [6]. This allows the sensitivity for muon pairs to be extended down to lower invariant masses. Muons with momentum less than $3.0 \mathrm{GeV} / c$ were required to have deposited energy in the ECAL equal to less than half their momentum, and to have fired more than $25 \%$ of the number of HCAL planes expected for a muon. If the muon's momentum is greater than $1.5 \mathrm{GeV} / c$ then the energy deposited in the HCAL must be greater than $80 \%$ of its momentum, otherwise it must be greater than $60 \%$ of its momentum.

The $\mathrm{d} E / \mathrm{d} x$ information for each track was employed to give the probability for each track to be a pion, kaon or proton. The product of these gives the probability for the pair of tracks to both be pions, kaons or protons. These are denoted as $P^{\left(\pi^{+}, \pi^{-}\right)}, P^{\left(\mathrm{K}^{+}, \mathrm{K}^{-}\right)}$and $P^{(\mathrm{p}, \overline{\mathrm{p}})}$ respectively.

A meson pair of type $a$ was defined to have

- $P^{\left(a^{+}, a^{-}\right)}>0.05$

- $P^{\left(b^{+}, b^{-}\right)}<0.05$

where $a=\pi, b=(\mathrm{K}, \mathrm{p})$ for pion pair selection, and $a=\mathrm{K}, b=(\pi, \mathrm{p})$ for kaon pair selection. For the kaon pair selection the invariant mass was recalculated using the kaon mass for each charged particle. Events with $W_{\gamma \gamma}$ greater than $6.0 \mathrm{GeV} / c^{2}$ were then rejected.

After studying a number of background sources, as described in section 6 , the following additional cuts were imposed. At least one of the tracks in the event must have a momentum such that the ionisation expected for a kaon 
differs from that expected for a pion by more than $14 \%$ of the value expected for a minimum ionising particle. The following cuts were applied only to the pion sample, which is particularly susceptible to backgrounds from both muon pairs and non exclusive hadron production:

- $\Sigma \mathbf{p}_{\mathrm{t}}<0.05 \mathrm{GeV} / c$;

- the angle between the tracks measured in the plane perpendicular to the beam direction must be greater than 3.13 radians;

- if a track has momentum less than $1.5 \mathrm{GeV} / c$, then any associated cluster in the electromagnetic calorimeter must have energy equal to less than $50 \%$ of its momentum, and any associated cluster in the hadron calorimeter must have energy equal to less than $10 \%$ of its momentum.

The final selection consisted of 165 kaon and 318 pion pair events.

\section{$5 \quad$ Trigger Efficiency}

The event selection described above was designed to select events in a region where the ALEPH trigger is known to be highly efficient. This analysis takes advantage of a specific ALEPH trigger which is fired when two approximately back to back tracks are seen in the tracking detectors. The efficiency of this trigger was measured using the process $\gamma \gamma \rightarrow \mathrm{e}^{+} \mathrm{e}^{-}$which is also independently triggered by energy deposits in the electromagnetic calorimeter. From this the efficiency curve was derived as a function of the invariant mass of the final state and used to correct the data. At invariant masses of $2.0 \mathrm{GeV} / c^{2}$ the efficiency was measured to be $95 \%$, rising to a value of $100 \%$ for invariant masses of $4.0 \mathrm{GeV} / c^{2}$ and above.

\section{Backgrounds}

Background from $\gamma \gamma \rightarrow \tau^{+} \tau^{-}$was estimated using the program described in [7], and that from $\gamma \gamma \rightarrow \mu^{+} \mu^{-}$using a program described in [8]. The background from $\tau$ pairs was found to be negligible but muon pairs are a significant background to the $\pi^{+} \pi^{-}$measurement.

The background to the pion pairs from misidentified kaon pairs was computed using the simulated kaon signal (see section 7). The normalisation was chosen to match the observed kaon signal. An equivalent procedure was used to find the background to the kaon pairs from misidentified pions. As 
this introduces a mutual dependance of the pion and kaon measurements on each other, the analysis was repeated until a stable result was obtained. This was achieved after five iterations.

The background from non exclusive $\gamma \gamma \rightarrow$ hadron production has been checked using the PHOJET program, which is known to give a good description of ALEPH untagged two photon physics events [9]. A small background of events was found in the $\pi^{+} \pi^{-}$sample, almost entirely due to events containing two pions plus undetected low energy photons. Overall there was an $18 \%$ background in the pion sample and an $11 \%$ background in the kaon sample. The significant backgrounds are listed in Tables 1 and 2.

\section{Calculation of Selection Efficiency}

The selection efficiencies for pion and kaon pairs were calculated using a version of the program of [7], which simulates the QED process $\mathrm{e}^{+} \mathrm{e}^{-} \rightarrow$ $\mathrm{e}^{+} \mathrm{e}^{-} \mathrm{l}^{+} \mathrm{l}^{-}$, modified in accordance with Eq. 1. The modification consisted initially of weighting events by $1 / W^{4}$. This set is referred to as 'Simulation A', and is shown in Fig 1. As can be seen the data sits slightly above the curve so two further sets, referred to as simulations $\mathrm{B}$ and $\mathrm{C}$ were generated with steeper dependence on $\cos \theta^{*}$ while still being consistent with the data. Set $B$ was weighted by an additional $1 / \sin ^{4} \theta^{*}$ term compared to set A while set $C$ was weighted by an additional $1 / \sin ^{6} \theta^{*}$ term. All three sets gave an acceptable description of the data. Simulation B was used to obtain the selection efficiencies as it best described the data. The other two sets were used in the calculation of systematic errors as described below.

The efficiencies are shown in Tables 3 and 4 . Three quarters of events are lost due to the boost of the final state, the remaining loss of efficiency is due to the tight cuts needed to remove background events.

\section{Systematic Uncertainties}

The chief source of systematic error in this analysis is the dependence on simulation to calculate selection efficiencies and backgrounds. This can be classified into two major components, firstly the dependence on accurate simulation of the detector, and secondly a dependence on the physics of the signal.

A number of critical elements of the detector simulation were studied. In all cases this was done by varying the aspect to be studied in the Monte Carlo sample and repeating the analysis. The dependence on the $\mathrm{d} E / \mathrm{d} x$ simulation 
was checked by slightly improving, and slightly degrading the resolution in a range that was still consistent with the data. The dependence on the momentum resolution was similarly obtained. To test for sensitivity to simulation of clusters and noise in the calorimeters the total energy in the event was increased and decreased by $50 \mathrm{MeV}$. The systematic error due to detector simulation was defined as half the difference between the largest and smallest value seen in a given bin as a result of these changes.

Concerning the dependence of the result on the simulation of the physics process, it is known that the angular dependence varies considerably at low masses [10], and this could cause changes to the calculated efficiencies. To test this the measurement was repeated with simulations $\mathrm{A}$ and $\mathrm{C}$, and the systematic error from this source was calculated as half the difference in efficiency between these two sets in each bin. The systematic error due to limited Monte Carlo statistics was also calculated. The accuracy of the measurement of the trigger efficiency is limited by the available statistics for the process $\gamma \gamma \rightarrow \mathrm{e}^{+} \mathrm{e}^{-}$. The systematic error on the trigger efficiency was estimated by studying the range of curves that were consistent with the data. In all cases this was found to be insignificant compared to the other systematic errors and has been neglected. All systematic errors are listed in Tables 3 and 4 . The total error was obtained by adding the systematic and statistical errors in quadrature.

\section{Measurement of the $\cos \theta^{*}$ Distribution}

The analysis is limited to the region $\left|\cos \theta^{*}\right|<0.6$ as the efficiency is acceptable in this region, and it matches the region used in the existing measurement by TPC/Two-Gamma [11]. The $\cos \theta^{*}$ distribution is calculated as follows. The differential cross section in a bin is given by

$$
\mathrm{d} \sigma / \mathrm{d} \cos \theta^{*}=\frac{N_{\text {data }}-N_{\text {back }}}{\epsilon \times L \times L_{\gamma \gamma} \times \delta \cos \theta^{*}}
$$

where $N_{\text {data }}$ is the number of events observed in a bin (weighted by the trigger correction), $N_{\text {back }}$ is the predicted number of background events in that bin, $\epsilon$ is the selection efficiency, $L$ is the integrated luminosity of the data, $L_{\gamma \gamma}$ is the luminosity function $\mathrm{d} L_{\gamma \gamma} / \mathrm{d} W_{\gamma \gamma}$ integrated over the range $2<W_{\gamma \gamma}<6$, and $\delta \cos \theta^{*}$ is the bin width. The luminosity function is the number which converts a cross section for a process $\mathrm{e}^{+} \mathrm{e}^{-} \rightarrow \mathrm{e}^{+} \mathrm{e}^{-} X$ to a cross section for the process $\gamma \gamma \rightarrow X$. It was calculated using the GALUGA program [12].

All measurements were performed independently for each year of data taking. The final results were taken as the weighted mean of all the 
Table 1: Significant backgrounds as a percentage of the signal in bins of $\cos \theta^{*}$.

\begin{tabular}{|c|c|c|c|c|c|}
\multicolumn{2}{c}{$\gamma \gamma \rightarrow \pi^{+} \pi^{-}$} & \multicolumn{2}{c}{$\gamma \gamma \rightarrow \mathrm{K}^{+} \mathrm{K}^{-}$} \\
\hline Bin $(\mathrm{GeV})$ & $\mathrm{K}^{+} \mathrm{K}^{-}$ & $\mu^{+} \mu^{-}$ \\
\hline $0.00-0.20$ & 5.2 & 3.0 \\
$0.20-0.40$ & 4.9 & 16.5 \\
$0.40-0.60$ & 3.7 & 23.1 \\
\hline
\end{tabular}$\quad$\begin{tabular}{|cc|c}
\hline $0.00-0.20$ & 4.9 \\
$0.20-0.40$ & 11.6 \\
$0.40-0.60$ & 9.0 \\
\hline
\end{tabular}

data, where the weighting is taken from the statistical errors. The overall systematic error was taken as the mean of the fractional errors measured in each year.

The result is shown in Fig. 1 and Table 3. The distributions are well described by the QCD prediction of $\mathrm{a}^{-4} \sin ^{-4} \theta^{*}$ dependence. They are also matched by the distribution in the signal Monte Carlo.

\section{Measurement of Invariant Mass Distribution}

The cross section for $\sigma\left(\gamma \gamma \rightarrow \mathrm{M}^{+} \mathrm{M}^{-}\right)$is calculated as a function of $W_{\gamma \gamma}$ using the formula:

$$
\sigma=\frac{\left(N_{\text {data }}-N_{\text {back }}\right) / \delta W_{\gamma \gamma}}{\epsilon \times L \times \mathrm{d} L_{\gamma \gamma} / \mathrm{d} W_{\gamma \gamma}}
$$

where $N_{\text {data }}$ is the number of events observed in a bin (weighted by the trigger correction), $N_{\text {back }}$ is the predicted number of background events in that bin, $\epsilon$ is the selection efficiency, $L$ is the integrated luminosity of the data, $\mathrm{d} L_{\gamma \gamma} / \mathrm{d} W_{\gamma \gamma}$ is the luminosity function, and $\delta W_{\gamma \gamma}$ is the bin width. The results are plotted in Fig. 2, and tabulated in Table 4. Also shown on Fig. 2 are results from TPC/Two-Gamma [11] and a leading twist QCD prediction. This was calculated as described using the formalism in [2] but, following a recommendation in [13], the pion distribution amplitude has been set to its asymptotic form, $\phi_{\mathrm{M}}=\sqrt{3} x(1-x)$. This leads to a prediction for the cross section for pion pair production of $100 \times \alpha_{s}^{2} / W^{6} \mathrm{nb}$, which for reasonable values of $\alpha_{s}$ is markedly smaller than the value predicted in [2]. The prediction for kaons is obtained from that for pions by multiplying it by 2.23 which is the ratio of the kaon and pion decay constants to the fourth power. The band of predictions shown in Fig. 2 was obtained by varying $\alpha_{s}$ from 0.3 to 0.6 . 

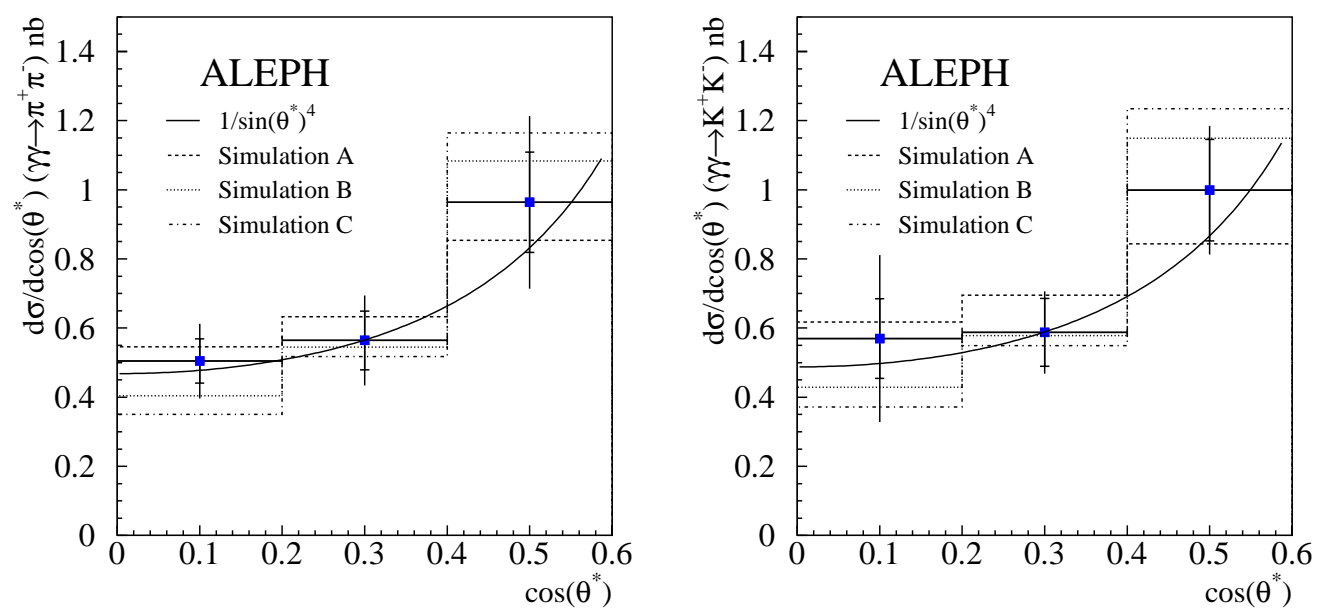

Figure 1: Measured distribution for $\cos \theta^{*}$ in $\gamma \gamma \rightarrow \pi^{+} \pi^{-}$(left) and $\gamma \gamma \rightarrow$ $\mathrm{K}^{+} \mathrm{K}^{-}$(right). The solid curve shows the shape of the QCD prediction. The histogram labeled 'Simulation B' is the distribution in the simulation used to calculate the efficiency. The histograms labeled $\mathrm{A}$ and $\mathrm{C}$ are the models used to estimate the model systematic error. The curve is normalised to match the central data point. The histograms are normalised to the data.

Both the pion and kaon data match the predicted shape well, but not the normalisation, particularly in the case of the pions. A function of the form $k / W^{6}$ was fitted to the pion and kaon data. This gave values for $k$ of $200 \pm 40 \mathrm{nb}\left(\mathrm{GeV} / \mathrm{c}^{2}\right)^{6}$ for pions $\left(\chi^{2}=1.9\right.$ for 7 degrees of freedom $)$ and $220 \pm 40 \mathrm{nb}\left(\mathrm{GeV} / \mathrm{c}^{2}\right)^{6}$ for kaons $\left(\chi^{2}=1.5\right.$ for 4 degrees of freedom).

\section{Conclusions}

The exclusive production of pion and kaon pairs has been measured at higher masses than previously achieved. The shapes of the distributions in $\cos \theta^{*}$ and $W_{\gamma \gamma}$ are in good agreement with QCD predictions. The normalisation of the observed signals is however in disagreement with the QCD predictions. The measurements are well described by a function of the form $k / W^{6}$ where $k=200 \pm 40 \mathrm{nb}\left(\mathrm{GeV} / \mathrm{c}^{2}\right)^{6}$ for pions and $k=220 \pm 40 \mathrm{nb}\left(\mathrm{GeV} / \mathrm{c}^{2}\right)^{6}$ for kaons. 

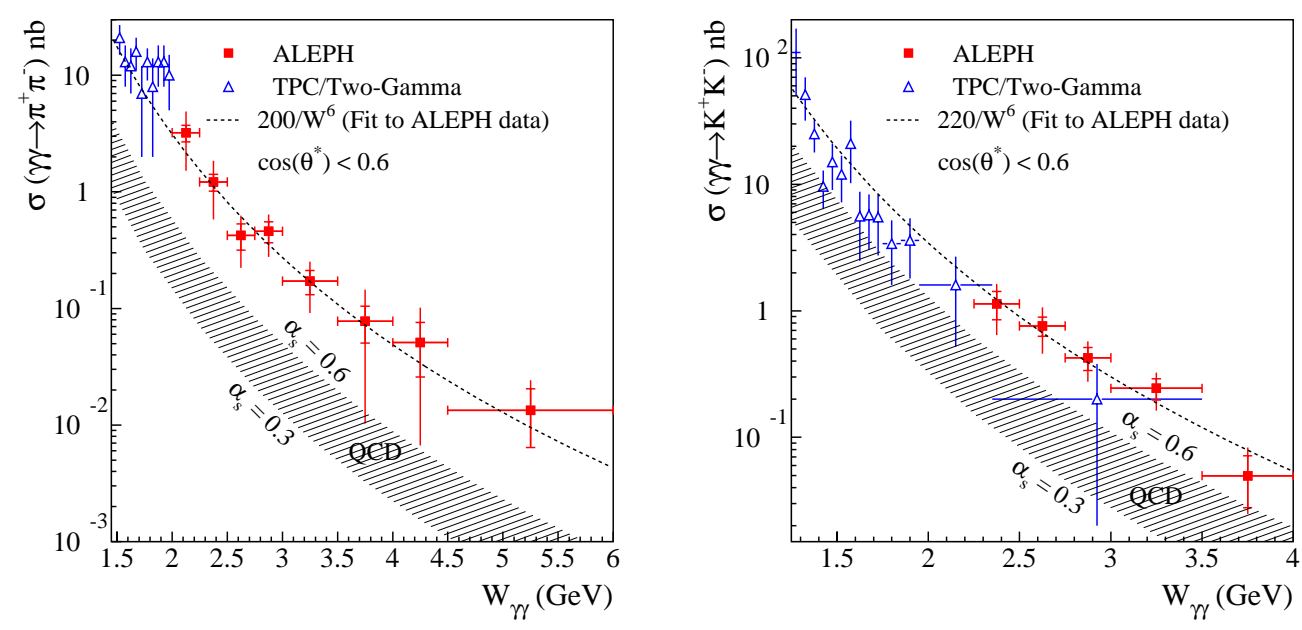

Figure 2: Measured distribution for $\gamma \gamma \rightarrow \pi^{+} \pi^{-}$(left) and $\gamma \gamma \rightarrow \mathrm{K}^{+} \mathrm{K}^{-}$ (right) as a function of $W_{\gamma \gamma}$. Also shown are results from TPC/TwoGamma [11], a leading twist QCD calculation. as described in the text, and the result of a fit to the ALEPH data.

Table 2: Significant backgrounds as a percentage of the signal in bins of $W_{\gamma \gamma}$.

\begin{tabular}{|c|c|c|c|c|}
\hline $\operatorname{Bin}(\mathrm{GeV})$ & $K^{+} K^{-}$ & $\mu^{+} \mu^{-}$ & \multirow{2}{*}{\multicolumn{2}{|c|}{$\gamma \gamma \rightarrow K^{+} K^{-}$}} \\
\hline $2.00-2.25$ & 5.4 & 4.9 & & \\
\hline $2.25-2.50$ & 5.0 & 16.0 & $\frac{5}{2525}$ & \\
\hline $2.50-2.75$ & 6.1 & 26.6 & $2.25-2.50$ & 7.8 \\
\hline $2.75-3.00$ & 3.1 & 16.9 & $2.50-2.75$ & 7.4 \\
\hline $3.00-3.50$ & 3.8 & 18.9 & $2.75-3.00$ & 7.5 \\
\hline $3.50-4.00$ & 2.9 & 00 & $3.00-3.50$ & 8.7 \\
\hline $4.00-4.50$ & 2.1 & 5.4 & $3.50-4.00$ & 16.2 \\
\hline $4.50-6.00$ & 2.2 & 0.0 & & \\
\hline
\end{tabular}


Table 3: $\mathrm{d} \sigma / \mathrm{d} \cos \theta^{*}$.

Pions

\begin{tabular}{|c|c|c|c|c|c|c|c|}
\hline $\begin{array}{c}\text { Bin } \\
(\mathrm{GeV})\end{array}$ & Efficiency & $\begin{array}{c}\text { Result } \\
(\mathrm{nb})\end{array}$ & \multicolumn{6}{|c|}{ Statistical } & \multicolumn{5}{|c|}{ Simulation } & M.C. stats. & \multicolumn{1}{c|}{ Model } & Total \\
\hline $0.00-0.20$ & 0.017 & 0.50 & 0.064 & 0.024 & 0.050 & 0.067 & 0.11 \\
$0.20-0.40$ & 0.013 & 0.56 & 0.085 & 0.031 & 0.053 & 0.078 & 0.13 \\
$0.40-0.60$ & $8.2 \times 10^{-3}$ & 0.96 & 0.14 & 0.076 & 0.080 & 0.17 & 0.25 \\
\hline
\end{tabular}

Kaons

\begin{tabular}{|c|c|c|c|c|c|c|c|}
\hline \multirow{2}{*}{$\begin{array}{c}\mathrm{Bin} \\
(\mathrm{GeV})\end{array}$} & \multirow[t]{2}{*}{ Efficiency } & \multirow{2}{*}{$\begin{array}{c}\text { Result } \\
\text { (nb) }\end{array}$} & \multicolumn{5}{|c|}{ Error (nb): } \\
\hline & & & Statistical & Simulation & M.C. stats. & Model & Total \\
\hline $0.00-0.20$ & $6.5 \times 10^{-3}$ & 0.57 & 0.12 & 0.14 & 0.13 & 0.083 & 0.24 \\
\hline $0.20-0.40$ & 0.011 & 0.59 & 0.098 & 0.023 & 0.052 & 0.036 & 0.12 \\
\hline $0.40-0.60$ & $7.1 \times 10^{-3}$ & 1.00 & 0.15 & 0.050 & 0.079 & 0.067 & 0.19 \\
\hline
\end{tabular}

Table 4: Table of results for cross section as a function of $W_{\gamma \gamma}$.

Pions

\begin{tabular}{|c|c|c|c|c|c|c|c|}
\hline $\begin{array}{c}\text { Bin } \\
(\mathrm{GeV})\end{array}$ & Efficiency & $\begin{array}{c}\text { Result } \\
(\mathrm{nb})\end{array}$ & \multicolumn{6}{|c|}{ Statistical } & \multicolumn{1}{|c|}{ Simulation } & \multicolumn{1}{c|}{ M.C. stats. } & \multicolumn{1}{c|}{ Model } & Total \\
\hline $2.00-2.25$ & $3.4 \times 10^{-3}$ & 3.21 & 0.52 & 0.36 & 0.85 & 1.32 & 1.69 \\
$2.25-2.50$ & 0.012 & 1.21 & 0.20 & 0.19 & 0.25 & 0.52 & 0.64 \\
$2.50-2.75$ & 0.020 & 0.42 & 0.11 & 0.095 & 0.098 & 0.10 & 0.20 \\
$2.75-3.00$ & 0.029 & 0.46 & 0.094 & 0.044 & 0.11 & 0.10 & 0.18 \\
$3.00-3.50$ & 0.033 & 0.17 & 0.040 & 0.012 & 0.046 & 0.050 & 0.080 \\
$3.50-4.00$ & 0.047 & 0.078 & 0.027 & 0.030 & 0.031 & 0.044 & 0.067 \\
$4.00-4.50$ & 0.021 & 0.051 & 0.025 & $8.2 \times 10^{-3}$ & 0.032 & 0.028 & 0.051 \\
$4.50-6.00$ & 0.061 & 0.013 & $7.0 \times 10^{-3}$ & $1.6 \times 10^{-3}$ & $5.7 \times 10^{-3}$ & $5.8 \times 10^{-3}$ & 0.011 \\
\hline
\end{tabular}

Kaons

\begin{tabular}{|c|c|c|c|c|c|c|c|}
\hline \multirow{2}{*}{$\begin{array}{c}\text { Bin } \\
(\mathrm{GeV})\end{array}$} & \multirow[t]{2}{*}{ Efficiency } & \multirow{2}{*}{$\begin{array}{c}\text { Result } \\
\text { (nb) }\end{array}$} & \multicolumn{5}{|c|}{ Error (nb): } \\
\hline & & & Statistical & Simulation & M.C. stats. & Model & Total \\
\hline $2.25-2.50$ & $5.8 \times 10^{-3}$ & 1.14 & 0.29 & 0.17 & 0.31 & 0.19 & 0.49 \\
\hline $2.50-2.75$ & 0.019 & 0.76 & 0.13 & 0.064 & 0.16 & 0.21 & 0.30 \\
\hline $2.75-3.00$ & 0.027 & 0.43 & 0.088 & 0.037 & 0.094 & 0.066 & 0.15 \\
\hline $3.00-3.50$ & 0.030 & 0.24 & 0.046 & 0.032 & 0.051 & 0.030 & 0.082 \\
\hline $3.50-4.00$ & 0.047 & 0.050 & 0.022 & 0.014 & 0.017 & 0.011 & 0.033 \\
\hline
\end{tabular}




\section{Acknowledgments}

We would like to thank our colleagues of the accelerator divisions at CERN for the outstanding performance of the LEP machine. Thanks are also due to the many engineers, and technical personnel at CERN and at the home institutes for their contribution to ALEPH's success. Those of us not from member states wish to thank CERN for its hospitality.

\section{References}

[1] S.J. Brodsky, "Two-Photon Exclusive Processes in $Q C D$ " in Proceedings of Photon 2000 (2001), ed. A. Finch, AIP Conference Proceedings Volume 571.

[2] S.J. Brodsky and G.P. Lepage, "Large Angle Two-Photon Exclusive Channels in Quantum Chromodynamics", Phys. Rev. D24 (1981) 1808.

[3] M. Benayoun and V.I. Chernyak, "SU(3)-Symmetry Breaking effects in $\gamma \gamma \rightarrow$ Two Mesons Processes", Nucl. Phys. B329 (1990) 285.

[4] M. Diehl, P. Kroll and C. Vogt, "The handbag contribution to $\gamma \gamma \rightarrow \pi \pi$ and $K K^{\prime \prime}$, Phys. Lett. B532 (2002) 99 ;

A. Szczurek and J. Speth, "Perturbative $Q C D$ versus pion exchange and hadronic FSI effects in the $\gamma \gamma \rightarrow \pi^{+} \pi^{-}$reaction", hep-ph/0207265.

[5] ALEPH Collaboration, "ALEPH: a detector for electron-positron annihilation at LEP", Nucl. Instrum. Meth. A294 (1990) 121;

ALEPH Collaboration, "Performance of the ALEPH detector at LEP", Nucl. Instrum. Meth. A360 (1995) 481;

B. Mours et al., "The design, construction and performance of the ALEPH silicon vertex detector", Nucl. Instrum. and Meth. A379 (1996) 101.

[6] C. Brew, "Measurement of the $F_{2}, F_{a}$ and $F_{b}$ Muonic Structure Functions of the Photon", Sheffield University Thesis, 1998.

[7] J.A.M. Vermaseren, "Two Photon Processes at Very High Energies", Nucl. Phys. B229 (1983) 347.

[8] F. A. Berends, P. H. Daverveldt and R. Kleiss, "Radiative Corrections To The Process $\mathrm{e}^{+} \mathrm{e}^{-} \rightarrow \mathrm{e}^{+} \mathrm{e}^{-} \mu^{+} \mu^{-"}$, Nucl. Phys. B253 (1985) 421. 
[9] P. Hodgson, "A Measurement of the Di-jet Cross-Sections in Two Photon Physics at LEP 2", Sheffield University Thesis, 2001.

[10] CLEO Collaboration, "Two photon production of charged pion and kaon pairs", Phys. Rev. D50 (1994) 3027.

[11] TPC/Two-Gamma Collaboration, "Pion and Kaon Pair Production in Photon-Photon Collisions", Phys. Rev. Lett. 57 (1986) 404.

[12] G.A. Schuler, "Two-photon physics with GALUGA 2.0", Computer Phys. Commun. 108 (1998) 279.

[13] M. Diehl, P. Kroll and C. Vogt, private communication. 